\title{
DESIGN OF A USB-BASED DATA ACQUISITION SYSTEM
}

\author{
Samiran Maiti \\ Asst. Professor, Department of Electronics \& Communication Engineering, Birbhum Institute of Engineering \& \\ Technology, Suri, Birbhum, West Bengal Pin-731101, samiran.cemk@gmail.com
}

\begin{abstract}
Development of a multi-channel compact data - acquisition board, which collects real world analog (0-5 Volt dc) and digital data, delivers them to a personal computer using a USB communication link. The DAQ board is based on microchip PIC microcontroller (PIC18F2550). A GUI is developed on Visual Basic which displays the instantaneous analog and digital data. It can also be used for generating the control action signal (manual) which could be delivered to the actuator through a DAC through the microcontroller.
\end{abstract}

Keywords: PIC Microcontroller, data acquisition, USB.

\section{DAS}

Data acquisition system, as the name implies, are products and/or processes used to collect information to document or analyze some phenomenon. Data acquisition is the sampling the real world to generate data that can be manipulated by a computer. Sometime abbreviated DAQ or DAS, data acquisition typically involves acquisition of signal waveforms and processing the signal to obtain desired information. the components of data acquisition system include appropriate sensors that convert any measurement parameter to an electrical signal, which is acquired by data acquisition hardware. Data acquisition begins with the physical phenomenon or physical property of an object (under investigation) to be measured. The physical property or phenomenon could be temperature change of a room, the intensity change of a light source, the pressure of a chamber, the force applied to an object or many other things. An effective data acquisition system can measure all of these properties or phenomenas.

\section{USB-BASED DATA ACQUISITION SYSTEM}

\subsection{Introduction}

With the introduction of low-power, fast performing, miniature embedded circuits in Instrumentation; data acquisition devices have become more compact, versatile and easy to use. Added with this, the computational power of digital computer is ever increasing, which has resulted data acquisition, presentation, processing and control operations to become more PC - centric. Small, compact DAQ devices, popularly known as DAS cards are very popular as frontend data collection units in all PC-based instrumentation systems. Primarily these cards offer analog conditioning functions (like filtering, isolation, excitation, amplification etc), followed by digitization of the signal. Finally, high-end microcontroller converts the digitized samples to a formatted data conforming a (serial) protocol for delivery to a desktop, laptop or even
PDA device. Universal Serial Bus (USB) has emerged as a very popular, inexpensive and easy-to-use short range $(5 \mathrm{~m})$ communication media for data transfer with a PC (host), where the communication device acts as a client. The USB was originally designed to connect PC with its peripherals (keyboard, mouse etc.). However, it has proven useful for many other applications, including measurement and automation. Use of Graphical User Interface (GUI) in Windows compatible application software have become an integral part of the software and are given equal importance with the algorithm development. GUI based system employ the use of graphical widgets like menu, edit box, pushbuttons, charts, displays etc to create an user friendly interface so that an inexperienced user can become quickly conversant with a complex applications.

The work is focused on the following objectives:

(i) To develop a PIC microcontroller based data acquisition card for collecting field data

(ii) To develop a USB communication link for bidirectional data and command transfer between a PC and the card

(iii) To develop a GUI, which acts as the user-end front panel to control entire operation of the system?

\subsection{Block Diagram:}

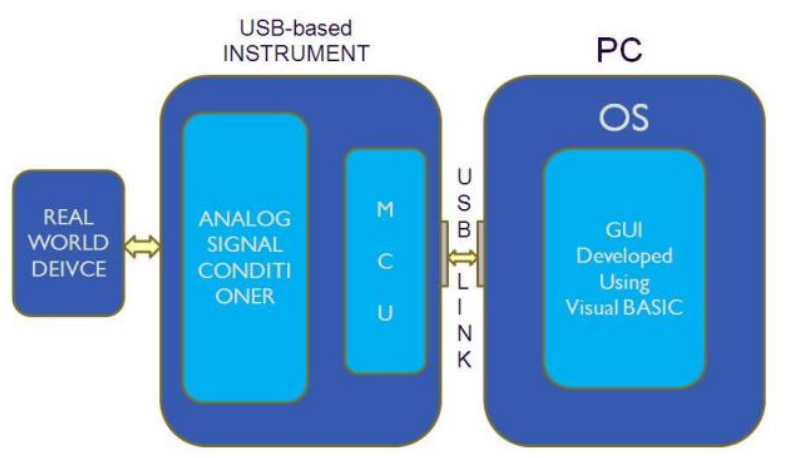




\subsection{Working}

There are two analog $\mathrm{i} / \mathrm{p}$ channels and 2 digital $\mathrm{i} / \mathrm{p}$ channels. Pin no. $2 \& 3$ of PIC (18F2550) Microcontroller are used for analog $\mathrm{i} / \mathrm{p} \&$ pin no. $11 \& 12$ are used for digital $\mathrm{i} / \mathrm{p}$. The analog $\mathrm{i} / \mathrm{p}$ range is from $0-5 \mathrm{v}$. Microcontroller's operating voltage, $\mathrm{VDD}=5 \mathrm{v}$ is given from the PC's USB port. The PIC (18F2550) Microcontroller has an inbuilt ADC of 10 bits. And the sampling frequency has been set at $50 \mathrm{~ms}$.

The first step is to write the program (firmware) for the PIC microcontroller. The program (firmware) is written in PicBasicPro language. The programming configures the USB descriptor so that the device is set under HID class. One of the benefits of a well-defined specification (like the USB HID class) is the availability of device drivers available in most modern operating systems.

The program is written in notepad (a software which is found in the accessories part of Windows XP) and is saved in .pbp format.

The program is compiled and converted it to HEX code before loading the program into the microcontroller's EPROM. Microcode Studio (front end) \& PicBasicPro header files (compilation engine) is used in this project for compiling the program (firmware). After compilation a HEX file is generated

This HEX file is burnt into the PIC microcontroller using WinPic800 software \& JDM programmer. This programmer (JDM) is a high voltage programmer, since low voltage programmers are not suitable for USB programming. The Winpic800 configuration bit settings needs to be configured properly. The proper configuration bit setting diagram is included.

The PIC18F2550 microcontroller can be connected to PC through USB port. As the device belongs to HID class so when it is connected to PC the operating software recognizes it as a HID device. One can check the properties of the device through USB View (a free tool from Microsoft Corporation) or Control Panel® Device Manager.

The GUI is developed using VB6 (Visual Basic 6) software. The GUI recognizes the USB device by its VID and PID when it is connected to a USB port. After the device is detected the user program can communicate with it. The flowcharts represent the program which have been written to create the GUI are included.

\subsection{Flow Chart}

\section{The flowchart of the firmware which has been loaded into the PIC18F2550}

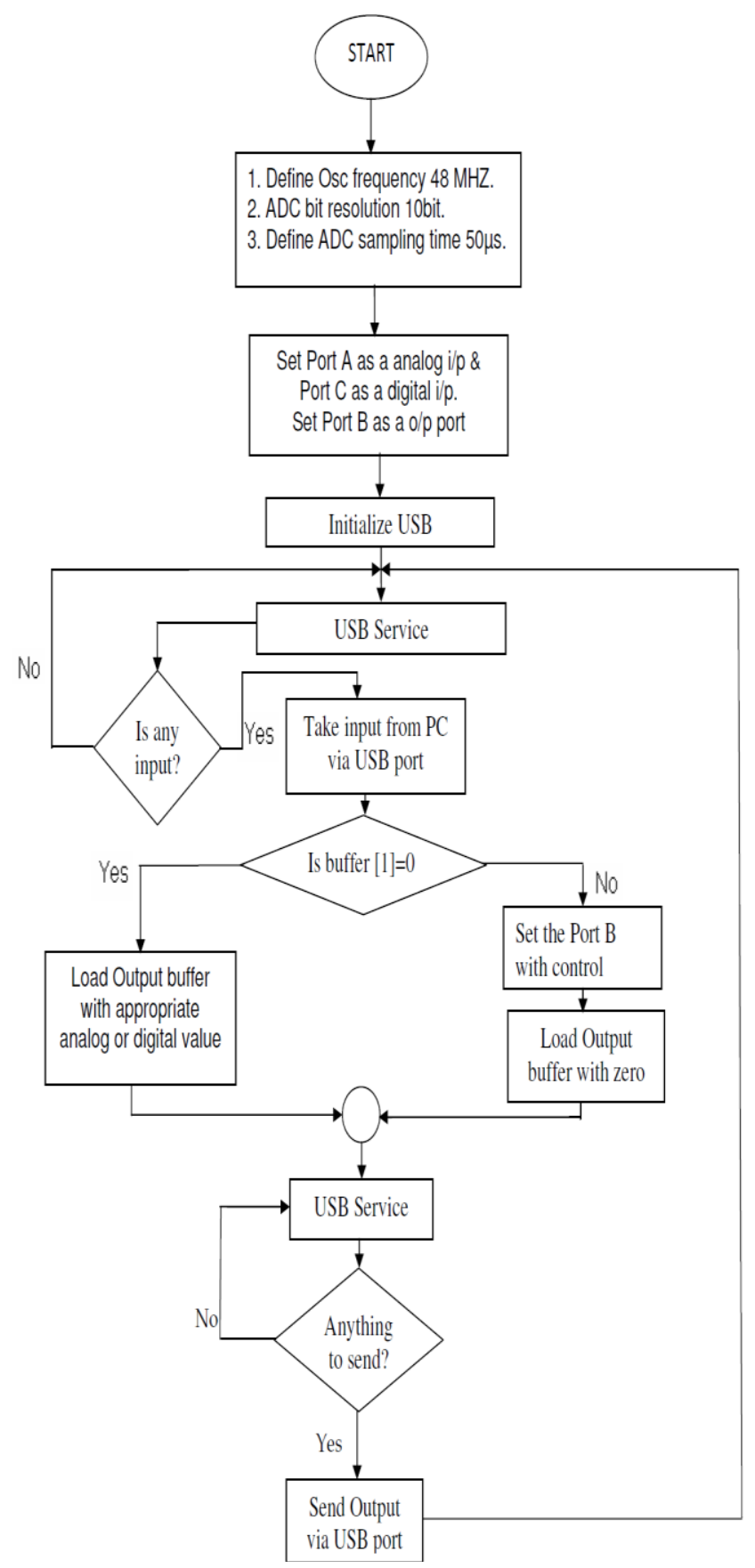

Flowchart of VB program to receive analog and digital channel values 


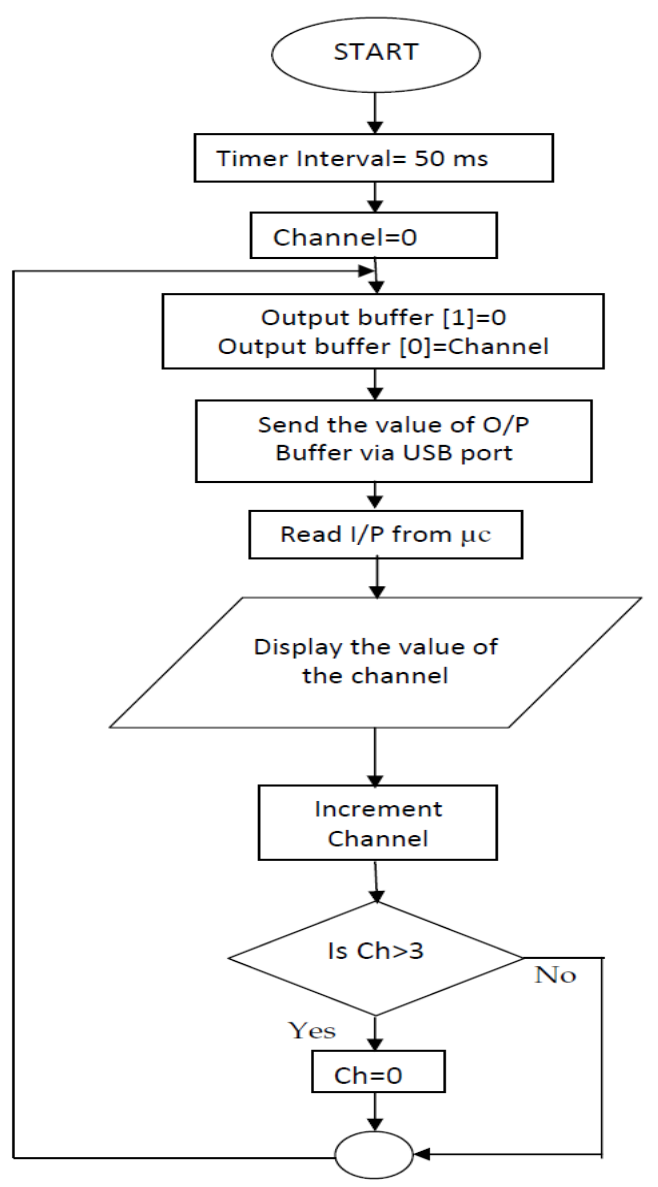

\section{Flowchart of VB program for control action} generation

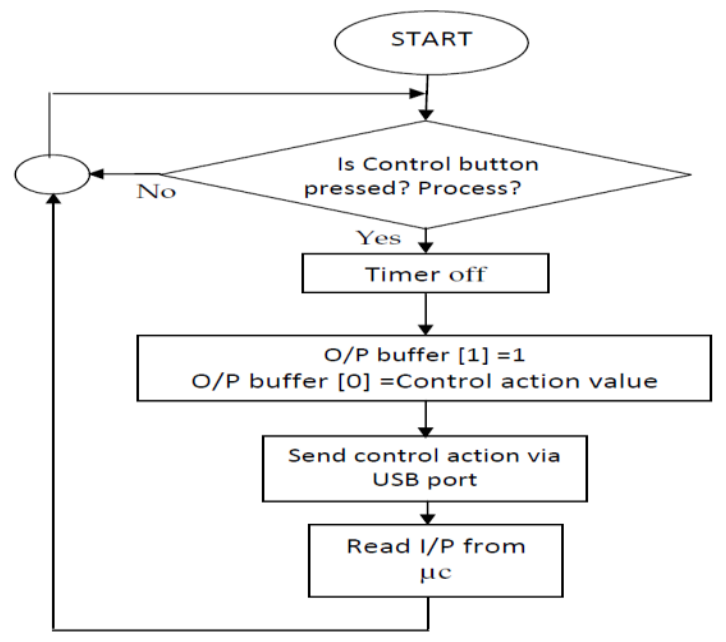

98SE. PIC 18F2550 Microcontroller itself have analog-todigital converter (ADC), digital-to-analog converter (DAC), timers and counters, serials ports, memory, input/output ports many more functional blocks on chip. So there is not required external analog-to-digital converter (ADC) and digital-toanalog converter (DAC). The system is reliable with the sensors. The system can be used as commercial product for temperature measurements and light intensity measurements. However, the existing system can be upgraded to a PC based data acquisition and control system, where, set-points along with controller tuning parameters can be provided using keyboard in the front panel GUI. In such a s case, the DAQ board shall include a DAC to deliver the control action signal (generated by the control algorithm developed either by microcontroller or at PC level) to the field.

The developed DAQ board accepts signal in $0-5 \mathrm{Vdc}$ level. For multi-channel and multi-sensor level acquisition, a separate sensor-specific analog conditioning board can be developed. Or, the same can be developed on the card itself, comprising with the small size modified board.

\section{REFERENCES}

[1] A. K. Sawhney, "A course in Electrical and Electronic Measurements and Instrumentation" Dhanpat Rai \& c.o.

[2] "USB Connectivity for Microcontrollers" Instrumentation Viewpoint, Sarti News Bulletin

[3] MPLAB IDE: www.microchip.com

[4] 2nd generation JDM PIC programmer and WinPic800 V3.6 foxdelta.com,

[5] USB Implementers Forum: USB 2.0 Specification: www.usb.org/developers/docs

[6] Data sheet PIC18F2550 Microcontroller.

\section{CONCLUSIONS}

This device scans the analog and digital channels at every $50 \mathrm{~ms}$ interval. No external power is necessary to run this device. As the device is of HID class it needs no device specific driver, even if the PC Operating system is Windows 